\title{
Primary testicular carcinoid tumour with mature teratoma in undescended testis metastatic to lymph nodes
}

\author{
Jae Heon Kim, MD; $;^{*}$ Tae II Noh, MD; Ji Sung Shim, MD; Byeong Kuk Ham, MD; Hoon Choi, MD; ${ }^{+}$ \\ Jae Hyun Bae, MD; Jae Young Park, MD
}

*Department of Urology, Soonchunhyang University College of Medicine, Soonchunhyang University Hospital, Seoul, Republic of Korea; †Department of Urology, Korea University College of Medicine, Korea University Ansan Hospital, Ansan, Republic of Korea

Cite as: Can Urol Assoc J 2014;8(3-4):e245-8. http://dx.doi.org/10.5489/cuai.383

Published online April 14, 2014.

\section{Abstract}

We report a case of primary testicular carcinoid tumour with mature teratoma metastatic to the para-aortic lymph node and the lymph node around the left gonadal vein, which was treated with radical orchiectomy, bleomycin, etoposide, and cisplatin chemotherapy, and modified retroperitoneal lymph node dissection. Three days after modified retroperitoneal lymph node dissection, bleomycin induced pneumonitis occurred, which was resolved with steroid administration. The patient is alive without recurrence 31 months after radical orchiectomy.

\section{Introduction}

A carcinoid tumour of the testis can be divided into 3 groups: primary carcinoid tumour, metastasis from a different location, and carcinoid tumour arising in a testicular teratoma. ${ }^{1}$ Cope was the first to report a testicular carcinoid tumour, a mestastasis from an ileal carcinoid tumour ${ }^{2}$ and Simon and colleagues reported the first primary testicular carcinoid tumour in $1954 .^{3}$ Although more than 60 cases have been reported to date, testicular carcinoid tumour still remains a very rare disease; moreover, a primary testicular carcinoid tumour with mature teratoma metastatic to the retroperitoneal lymph nodes is extremely rare. ${ }^{4}$ Because there was no effective treatment for the metastatic lesions, the prognosis for these cases have been disappointing. ${ }^{1}$

We report a primary testicular carcinoid tumour with lymph node metastasis which was cured by surgical resection and chemotherapy. Until now, only 8 cases metastatic to the lymph nodes or distant organs have been reported. ${ }^{4}$ To our knowledge, this is the first case of primary testicular carcinoid tumour occurring in the undescended testis with lymph node metastasis that was cured with surgical therapy and chemotherapy even though bleomycin-induced pneumonitis (BIP) developed.

\section{Case report}

A 47 year-old man presented to our urologic outpatient clinic with complaints of palpable mass in the left inguinal area. He had a history of untreated left cryptorchidism and viral B hepatitis. Among the serologic markers, serum lactate dehydrogenase (LDH) level was elevated to $798 \mathrm{IU} / \mathrm{L}$ and the levels of other tumour markers were within the normal range. Computed tomography (CT) demonstrated welldefined heterogeneous enhancing left intratesticular mass with internal tiny calcifications in the inguinal area (Fig. 1 , part A), an enlarged lymph node at the para-aortic area (3.5 cm in size) and the anterior aspect of left psoas muscle $(1.5 \mathrm{~cm}$ in size) (Fig. 1, part B and part C). Left radical orchiectomy was performed. Pathological examination showed testicular carcinoid tumour measuring $4.5 \times 3.2 \mathrm{~cm}$, extending to the peritesticular soft tissue. We also found mature teratoma in the focal area of the carcinoid tumour measuring $1.0 \times 0.8 \mathrm{~cm}$, confined to the testis, with free resection margin of the spermatic cord (Fig. 2). Immunohistochemistry in the carcinoid tumour revealed positive in cystokeratin, chromogranin A, synaptophysin, CD56, and negative in vimentin. Endoscopic examination of gastrointestinal tract did not show any primary carcinoid tumour.

Three courses of combination chemotherapy consisting of bleomycin, etoposide, and cisplatin (BEP) were administered to the patient. During chemotherapy, mild leukocytopenia and skin colour change emerged as complications. Followup positron emission tomography (PET) showed the enlarged lymph nodes in the paraaortic and psoas muscle area with mild to moderate hypermetabolism (Fig. 3). The serum LDH level was above the normal range after BEP chemotherapy. Considering these clinical findings, including abnormal PET results and elevated $\mathrm{LDH}$ level, modified retroperitoneal 


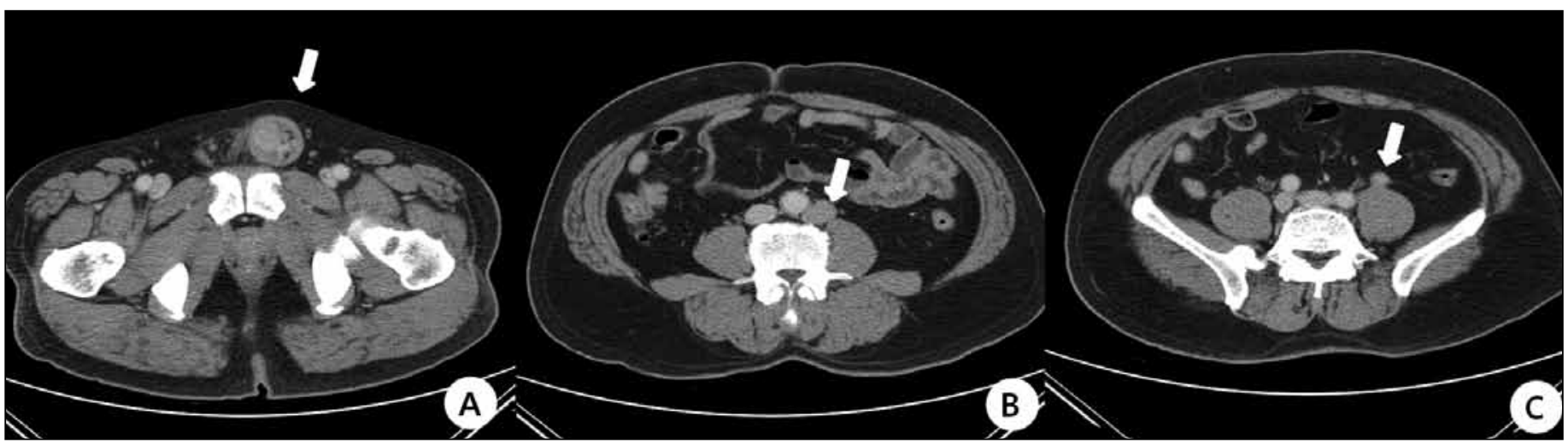

Fig. 1. (A) Well defined heterogeneous enhancing left intratesticular mass (white arrow) with internal tiny calcifications in the inguinal area, (B) enlarged lymph node at the para-aortic area (white arrow), and $(C)$ enlarged lymph node with a size of $1.5 \mathrm{~cm}$ (white arrow) at the anterior aspect of the left psoas muscle.

lymph node dissection (mRPLND) was performed and the metastatic lesions were resected with free surgical margins. Three days after mRPLND, the patient suddenly complained of respiratory distress which was BIP as shown through a chest CT. For 3 days, the patient underwent medical support in the intensive care unit due to acute pulmonary distress; he recovered after steroid administration. The surgical specimen from mRPLND did not include teratomatous component and showed the same histology as previous primary testicular carcinoid tumour (Fig. 4).

At 4 months after mRPLND, serum LDH level returned to the normal range and 24 urine 5-hydroxyindoleacetic acid level showed a normal level of $3.1 \mathrm{mg} /$ day. At 8 months after mRPLND, abdominal CT scan showed no recurrence of any suspicious metastatic lesions (Fig. 5). The patient is alive without recurrence 31 months after radical orchiectomy with complaint of a slightly reduced ejaculatory volume without erectile dysfunction.

\section{Discussion}

Carcinoid tumours originate from neuroendocrine cells and thus can have endocrine activity. Among the carcinoid tumours, $85 \%$ occur in the gastrointestinal tract, mostly in the ileum and appendix, and other sites, including the lungs, pancreas, biliary tract, thymus, ovary and rarely testis. 5,6 Our case belongs to a primary carcinoid tumour arising in a testicular teratoma. Although the size of the carcinoid tumour was larger than that of the mature teratoma in our case, the origin of the testicular carcinoid tumour is postulated to be a one-sided development of the mature teratoma or differentiation of toti-potential germ cells in the testis to argentaffin-like cells. ${ }^{7}$

Vasoactive products produced by the tumour can cause carcinoid syndrome, which is characterized by sweating, flushing, wheezing, diarrhea, abdominal pain, cardiac valvular fibrosis and pellagra dermatosis. ${ }^{5}$ Carcinoid syndrome occurs in $8 \%$ of patients with a carcinoid tumour,

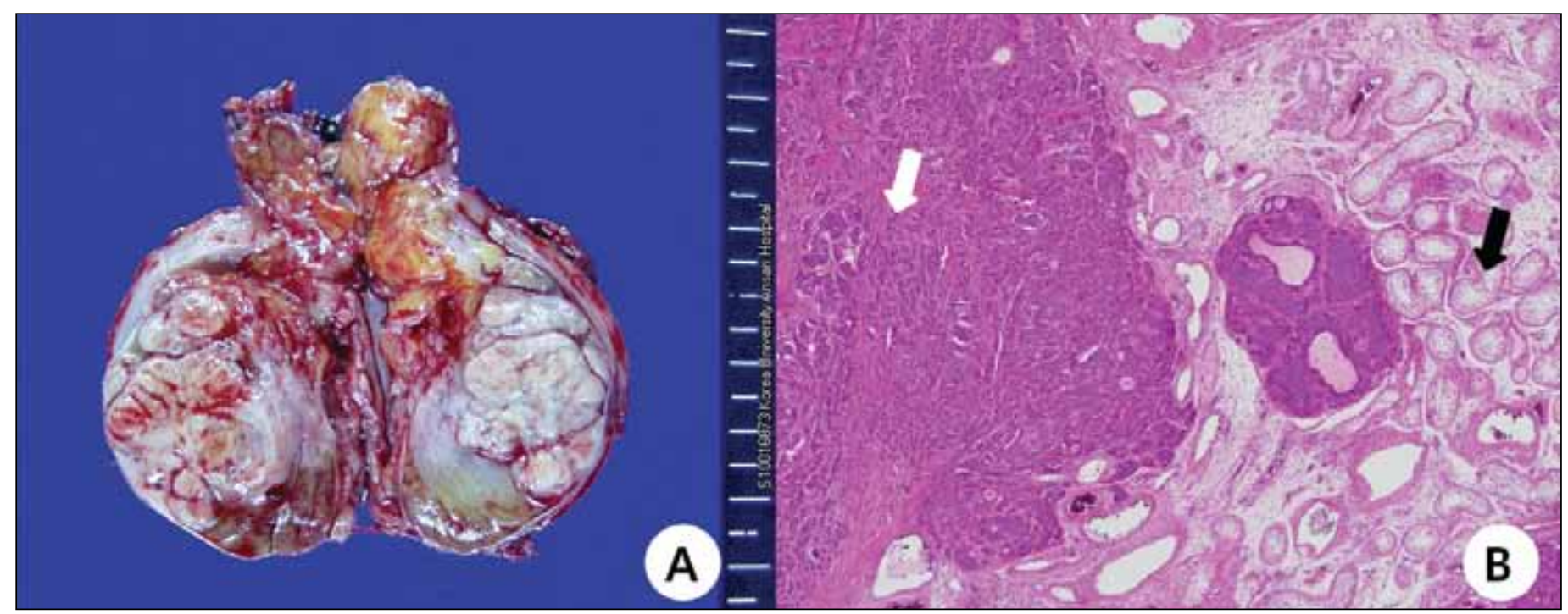

Fig. 2. (A) Macroscopic appearance of the testicular carcinoid tumor. (B) Microscopic appearance of the testicular carcinoid tumor with typical rosette formation (white arrow) and mature teratoma (black arrow) (hematoxylin and eosin stain $\times 100)$. 


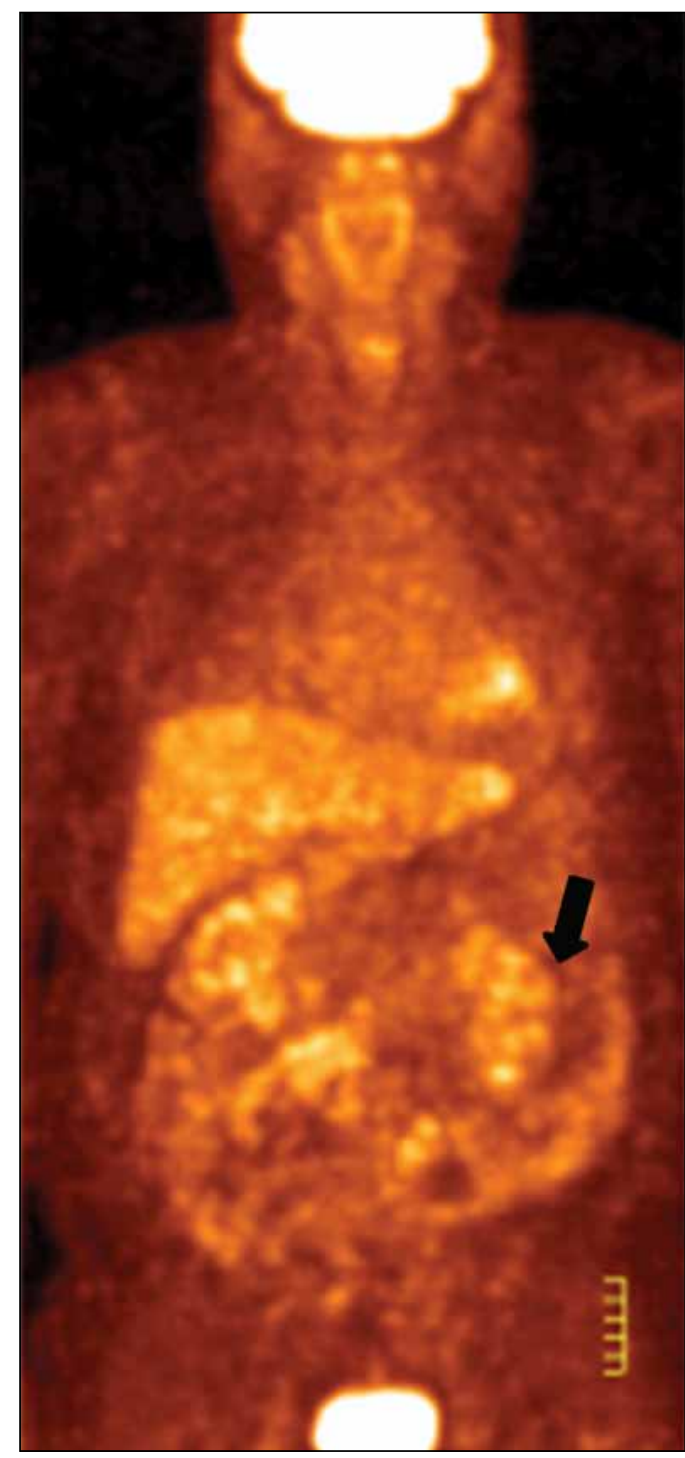

Fig. 3. Positron emission tomography showed the enlarged lymph nodes in the paraaortic and the psoas muscle area with mild to moderate hypermetabolism (black arrow).

especially in cases with metastasis, but our patient did not complain of any symptoms of carcinoid syndrome.

Carcinoid tumours were initially considered to be benign, but recently it has been considered to have malignant potential. ${ }^{8}$ Histologically, it is difficult to differentiate between benign and malignant carcinoid tumours. Therefore, it is important to evaluate the presence of metastases. Previous studies have reported that metastasis occurs in $11.6 \%$ of primay testicular carcinoid tumours and the frequency was less than that of intestinal carcinoid tumours. ${ }^{9}$ The independent predicting factors that have been suggested for metastases are the size of the primary tumour $(>7.3 \mathrm{~cm})$, poor diffrentiation of the tumours, and the presence of carcinoid syndrome. ${ }^{10}$ However, our patient included none of the predicting factors; his primary tumour was $4.5 \mathrm{~cm}$, the differentiation was not poor and typical rosette formation was observed; moreover, no symptoms of carcinoid syndrome were observed.

Surgery is the only way to diagnose testicular carcinoid tumours because it is almost impossible to differentiate carcinoid tumours from germ cell tumours in radiologic findings. ${ }^{1}$ No standard treatment has been established for metastatic testicular carcinoid tumours. In genereal, carcinoid tumours do not respond well to chemotherapy. ${ }^{11}$ The current combination chemothepary, which include streptozocin, 5-FU, cyclophosphamide or doxorubicin, has only genereated short-lasting response in fewer than $10 \%$ of patients. ${ }^{11,12}$ As for testicular carcinoid tumours, the regimens of chemotherapy have not been established and the prognosis of most patients with metastases has been discouraging. Radiotherapy also has little effect on carcinoid tumours. . $^{6,11,12}$

\section{Conclusion}

In the current case, we tried BEP combination chemotherpy for the metastatic lesion, however it remained unchanged. After mRPLND, the LDH level became normal and no evidence of disease recurrence was detected until the last follow-up. If the metastatic lesion is resectable, surgery might be the only potentially curative treatment. ${ }^{13}$ Our case revealed no recurrence of tumour during 31 months of follow-up. However, carcinoid tumours need long- term follow-up, because they have been known to cause metastases up to 7 years after the primary treatment. ${ }^{14}$

Competing interests: None declared.

This paper has been peer-reviewed.

Acknowledgement: This work was supported by the National Research Foundation of Korea (NRF) grant funded by the Korea government (MSIP) (No.2011-0020128). This work was also supported by the Soonchunhyang University Research Fund.

\section{References}

1. Stroosma OB, Delaere KP. Carcinoid tumours of the testis. BJU Int 2008;101:1101-5.

2. Cope Z. Metastasis of an argentaffin carcinoma in the testicle. $B r J$ Urol 1930;2:268-72. http://dx.doi. org/10.1111/i.1464-410X.1930.tb00005.x.

3. Simon HB, Mc DJ, Culp OS. Argentaffin tumor (carcinoid) occurring in a benign cystic teratoma of the testicle. J Urol 1954;72:892-4.

4. Fujita K, Wada R, Sakurai T, et al. Primary carcinoid tumor of the testis with teratoma metastatic to the para-aortic lymph node. Int J Urol 2005;12:328-31.

5. Soga J, Yakuwa Y, Osaka M. Carcinoid syndrome: a statistical evaluation of 748 reported cases. J Exp Clin Cancer Res 1999;18:133-41. 
Kim et al.

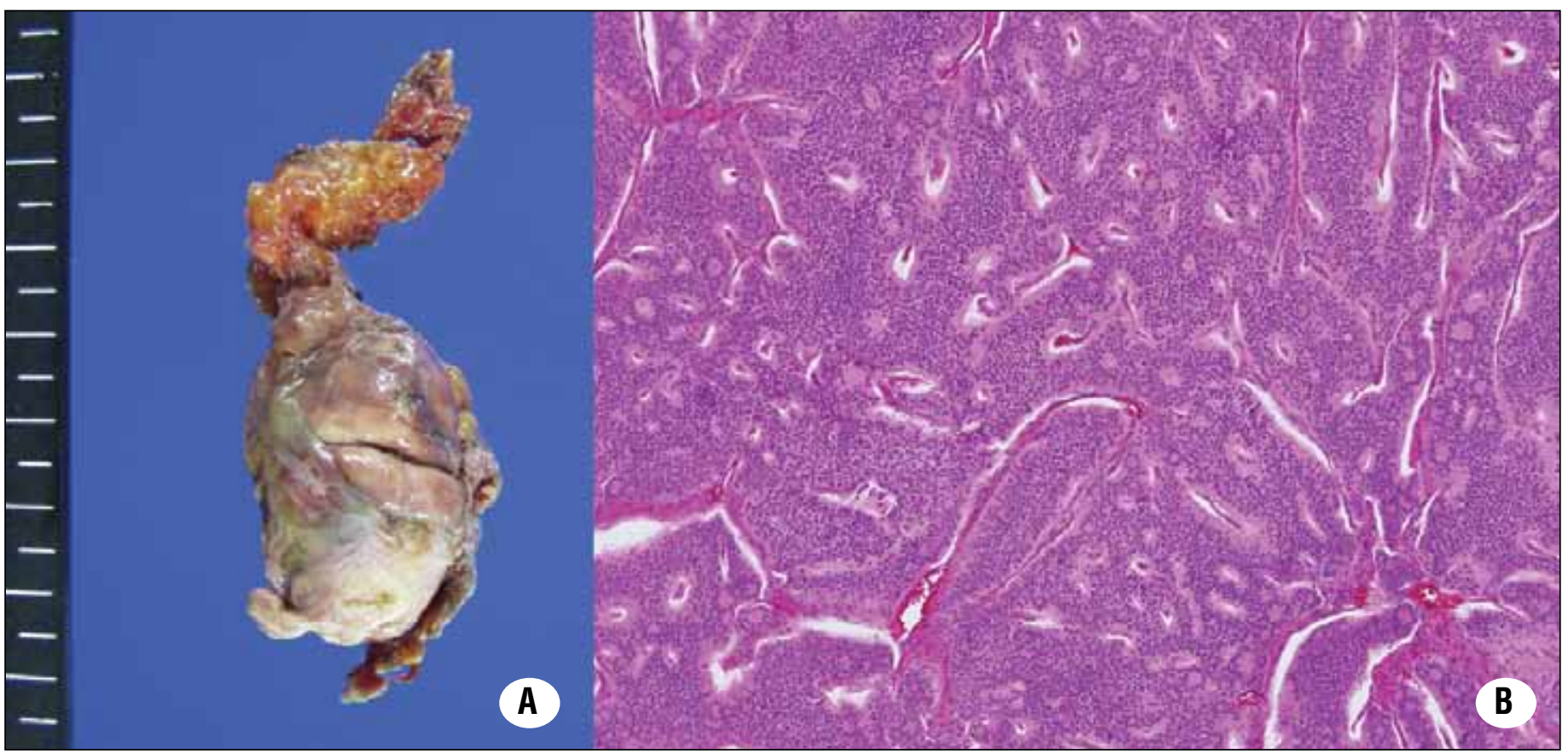

Fig. 4. (A) Macroscopic apperance of the metastatic para-aortic lymph node. (B) Microscopic appreance of the metastatic carcinoid tumor with typical rosette formation without teratoma component (hematoxylin and eosin stain $\times 100$ ).

6. Modlin IM, Latich I, Kidd M, et al. Therapeutic options for gastrointestinal carcinoids. Clin Gastroenterol Hepatol 2006;4:526-47.

7. Berdij CC, Mostofi FK. Carcinoid tumors of the testis. J Urol 1977;118:777-82.

8. Soga J. Carcinoids and their variant endocrinomas. An analysis of 11842 reported cases. J Exp Clin Cancer Res 2003;22:517-30.

9. Zavala-Pompa A, Ro JY, el-Naggar A, et al. Primary carcinoid tumor of testis. Immunohistochemical, ultrastructural, and DNA flow cytometric study of three cases with a review of the literature. Cancer 1993;72:1726-32.

10. Hayashi T, lida S, Taguchi J, et al. Primary carcinoid of the testis associated with carcinoid syndrome. Int J Urol 2001:8:522-4.

11. Oberg K. Chemotherapy and biotherapy in the treatment of neuroendocrine tumours. Ann Oncol 2001;12:S111-4.
12. Zuetenhorst JM, Taal BG. Metastatic carcinoid tumors: a clinical review. Oncologist 2005;10:123-31.

13. Sutherland RS, Wettlaufer JN, Miller GJ. Primary carcinoid tumor of the testicle: a case report and management schema. J Urol 1992;148:880-2.

14. Romero FR, Rais-Bahrami S, Permpongkosol S, et al. Primary carcinoid tumors of the kidney. J Urol 2006;176(6 Pt 1):2359-66.

Correspondence: Jae Young Park, Department of Urology, Korea University College of Medicine, Korea University Ansan Hospital, 123 Jeokgeum-ro, Danwon-Ku, Ansan-Si, Gyeonggi-Do, zip 425707, Republic of Korea; fax: 82-31-412-2370; jaeyoungpark@korea.ac.kr

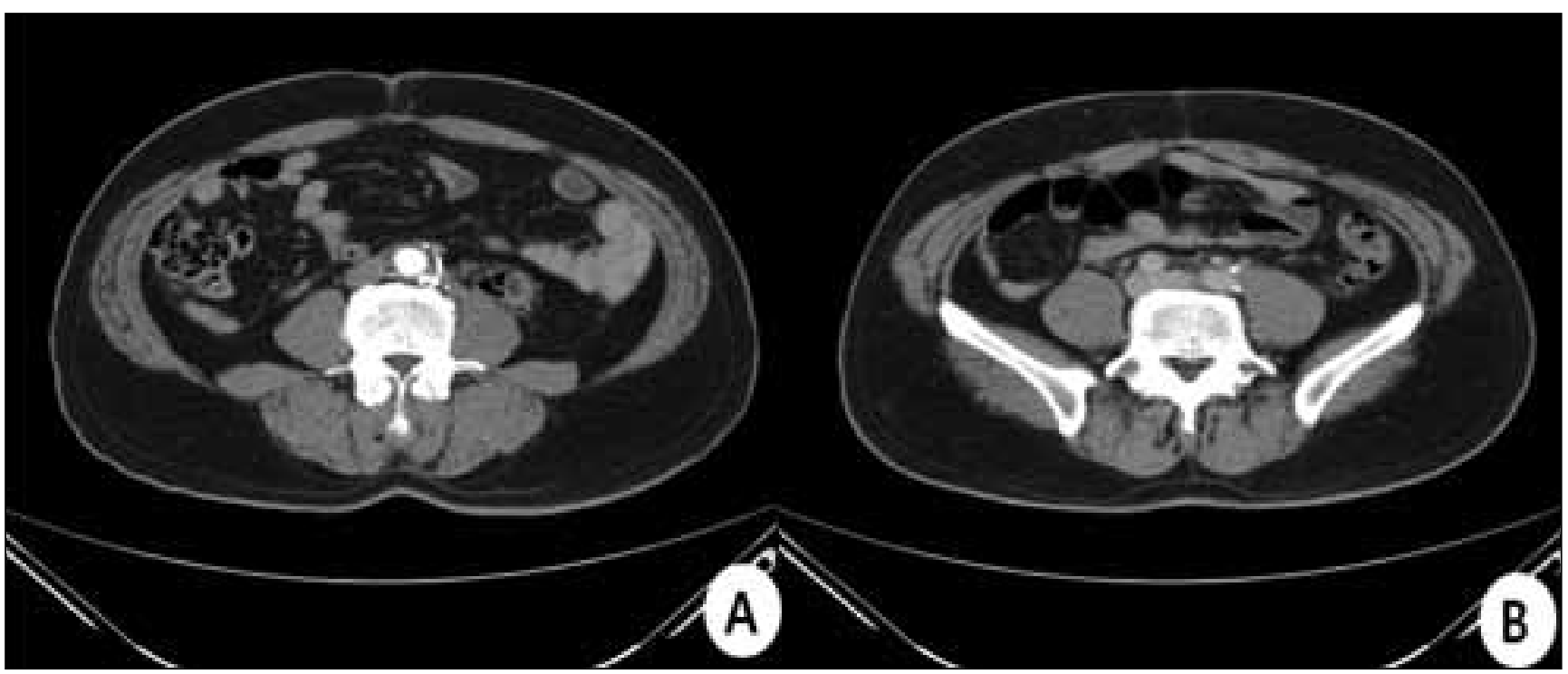

Fig. 5. No recurrence or residual metastatic tumor in follow-up computed tomography. 